TAIWANESE JOURNAL OF MATHEMATICS

Vol. 13, No. 2B, pp. 821-836, April 2009

This paper is available online at http://www.tjm.nsysu.edu.tw/

\title{
CONNECTEDNESS AND PATH-CONNECTEDNESS OF SOLUTION SETS TO SYMMETRIC VECTOR EQUILIBRIUM PROBLEMS
}

\author{
Ren-you Zhong, Nan-jing Huang and Mu-Ming Wong*
}

\begin{abstract}
In this paper, we study the connectedness and path-connectedness of the solution sets for symmetric vector equilibrium problems in locally convex Hausdorff topological vector spaces under some suitable assumptions. The results presented in this paper generalize some known results in $[10,14,24$, $32,33]$.
\end{abstract}

\section{INTRODUCTION}

In 1980, vector variational inequality was first introduced and studied by Giannessi [21]. Later on, vector variational inequality and its various extensions have been studied by Chen and Cheng [11], Chen, Huang and Yang [12], Yang [36] and other authors. Inspired by the study of vector variational inequalities, more general equilibrium problems (see [7, 8]) have been extended to the case of vector-valued bifunctions, known as vector equilibrium problems. The equilibrium problem contains as special cases, for instance, optimization problem, Nash equilibria problem, fixed point problem, variational inequalities, and complementarity problem (see, for example, $[1,2,9,13,22,26,27]$ and the references therein).

The system of vector equilibrium problems, i.e., a family of equilibrium problems for vector-valued bifunctions defined on a product set were introduced by Ansari et al. [3] with applications in vector optimization problems and Nash equilibrium problem for vector-valued functions. The system of vector equilibrium problems contain system of equilibrium problems, systems of vector variational inequalities,

Received December 30, 2008.

2000 Mathematics Subject Classification: 49J27, 49J40.

Key words and phrases: Symmetric vector equilibrium problem, $C$-convex, $C$-quasiconvex, $C$ concave, Connectedness, Path-connectedness.

This work was supported by the National Natural Science Foundation of China $(10671135,70831005)$ and Specialized Research Fund for the Doctoral Program of Higher Education (20060610005).

*Corresponding author. 
system of vector variational-like inequalities, system of optimization problems, fixed point problems and several related topics as special cases (see, for example, $[3,4$, $5,15,16,28,30,31,29,34]$ and the references therein).

On the other hand, the symmetric vector equilibrium problem which is a generalization of the equilibrium problem has been studied by many authors. A main topic of current research is to establish existence theorems (see, for example, [18, $19,20,23])$. Another important topic is to study the topological properties of the solution sets, as it provides the possibility of continuously moving from one solution to any other solution.

Recently, Cheng [14] and Lee ea al. [32] studied the connectedness of weak efficient solutions set for single-valued vector variational inequalities in finite dimensional Euclidean space. Lee and $\mathrm{Bu}$ [33] studied the connectedness of the solution sets for affine vector variational inequalities with noncompact polyhedral constraint sets and positive semidefinite (or monotone) matrices. Gong [24] studied the connectedness and path-connectedness of various solution sets for single-valued vector equilibrium problems in a real locally convex Hausdorff topological vector space. Gong [25] studied the connectedness of the set of efficient solutions for the generalized system with monotone bifunctions in real locally convex Hausdorff topological vector spaces. Very recently, Chen et al. [10] studied the existence, connectedness, and the compactness of the weak efficient solutions set for set-valued vector equilibrium problems and the set-valued vector Hartman-Stampacchia variational inequalities in normed linear spaces. However, to the best of our knowledge, there is no paper dealing with the connectedness and path-connectedness of the solution set for the symmetric vector equilibrium problem.

Inspired and motivated by the works mentioned above, in this paper, we study the connectedness and path-connectedness properties of the solution set to the symmetric vector equilibrium problem in locally convex Hausdorff topological vector spaces. The results presented in this paper generalize some corresponding results in the literature $[10,14,24,32,33]$.

\section{Preliminaries}

Throughout this paper, let $X, Y, E$ and $Z$ be real locally convex Hausdorff topological vector spaces. Let $A \subset X$ and $B \subset E$ be nonempty closed convex subsets, $F: A \times B \times A \rightarrow 2^{Y}$ and $G: A \times B \times B \rightarrow 2^{Z}$ be two set-valued mappings. Let $C \subset Y$ and $P \subset Z$ be two closed convex pointed cones with int $C \neq \emptyset$ and int $P \neq \emptyset$. Let $Y^{*}$ and $Z^{*}$ be the topological dual spaces of $Y$ and $Z$, respectively. Let $C^{*}$ and $P^{*}$ be the dual cones of $C$ and $P$, respectively, that is,

$$
C^{*}=\left\{f \in Y^{*}:\langle f, y\rangle \geq 0, \text { for all } y \in C\right\}
$$


and

$$
P^{*}=\left\{g \in Y^{*}:\langle g, y\rangle \geq 0, \text { for all } y \in P\right\} .
$$

The symmetric vector equilibrium problem $(S V E P)$ is the problem of finding $(x, y) \in A \times B$ such that

$$
\begin{cases}F(x, y, u) \cap(-i n t C)=\emptyset, & \forall u \in A, \\ G(x, y, v) \cap(-i n t P)=\emptyset, & \forall v \in B\end{cases}
$$

Remark 2.1. (i) If $C=P, f: A \times B \rightarrow Y$ and $g: A \times B \rightarrow Z$ are two single-valued mappings,

$$
F(x, y, u)=f(u, y)-f(x, y), \quad \forall(x, y, u) \in A \times B \times A
$$

and

$$
G(x, y, v)=g(x, v)-g(x, y), \quad \forall(x, y, v) \in A \times B \times B,
$$

then (2.1) reduces to a single-valued symmetric vector equilibrium problem considered by Fu [18]; (ii) If $G \equiv 0, F(x, y, u)=f(x, u)$ for any $(x, y, u) \in A \times B \times A$, then (2.1) is the equilibrium problem which was considered and studied by many authors (see, for example, $[1,2,9,13,22,26,27]$ and the references therein); (iii) If $G \equiv 0, T$ is a mapping from $A$ to $L(X, Y)$ where $L(X, Y)$ denotes the space of all continuous linear operators from $\mathrm{X}$ to $\mathrm{Y}$, and $F(x, y, u)=\langle T x, u-x\rangle$ for any $(x, y, u) \in A \times B \times A$, then (2.1) is the classic vector variational inequality problem which was introduced by Giannessi [21].

For any $(f, g) \in\left(C^{*} \backslash\{0\}\right) \times\left(P^{*} \backslash\{0\}\right)$, we also consider the following scalar symmetric equilibrium problem $(S S E P)_{f, g}$ : finding $(x, y) \in A \times B$, such that

$$
\left\{\begin{array}{l}
f(F(x, y, u)) \geq 0, \quad \forall u \in A, \\
g(G(x, y, v)) \geq 0, \quad \forall v \in B .
\end{array}\right.
$$

We denote the solution sets of $(S V E P)$ and $(S S E P)_{f, g}$ by $S(F, G)$ and $S(f, g)$, respectively.

Definition 2.1. A set-valued mapping $T: X \rightarrow 2^{Y}$ is said to be

(i) closed if $\operatorname{GraphT}=\{(x, y) \in X \times Y: y \in T(x)\}$ is closed in $X \times Y$;

(ii) upper semicontinuous if, for every $x \in X$ and every open set $V$ satisfying $T(x) \subset V$, there exists a neighborhood $U$ of $x$ such that $T(U) \subset V$;

(iii) lower semicontinuous if, for any $x \in X, y \in T(x)$ and any neighborhood $\mathcal{N}(y)$ of $y$, there exists a neighborhood $\mathcal{N}(x)$ of $x$ such that $T(x) \bigcap \mathcal{N}(y) \neq \emptyset$ for all $x \in \mathcal{N}(x)$. 
Note that $T: X \rightarrow 2^{Y}$ is lower semicontinuous at $x \in X$ if and only if, for any sequence $x_{n} \rightarrow x$ and $y \in T(x)$, there exists a sequence $y_{n} \in T\left(x_{n}\right)$ such that $y_{n} \rightarrow y$.

Definition 2.2. Let $T: A \times B \times D \rightarrow 2^{Y}$ be a set-valued mapping. For any fixed $(x, y) \in A \times B, T(x, y, \cdot)$ is said to be

(i) $C$-convex if, for every $z_{1}, z_{2} \in D$ and $t \in[0,1]$,

$$
t T\left(x, y, z_{1}\right)+(1-t) T\left(x, y, z_{2}\right) \subset T\left(x, y, t z_{1}+(1-t) z_{2}\right)+C ;
$$

(ii) $C$-quasiconvex if, for every $z_{1}, z_{2} \in D$ and $t \in[0,1]$, either

$$
T\left(x, y, z_{1}\right) \subset T\left(x, y, t z_{1}+(1-t) z_{2}\right)+C
$$

or

$$
T\left(x, y, z_{2}\right) \subset T\left(x, y, t z_{1}+(1-t) z_{2}\right)+C .
$$

Definition 2.3. Let $T: A \times B \times D \rightarrow 2^{Y}$ be a set-valued mapping. For each fixed $z \in D, T(\cdot, \cdot, z)$ is said to be $C$-concave on $A \times B$ if, for any $\left(x_{1}, y_{1}\right),\left(x_{2}, y_{2}\right) \in A \times B$ and $t \in[0,1]$, we have

$$
T\left(t x_{1}+(1-t) x_{2}, t y_{1}+(1-t) y_{2}, z\right) \subset t T\left(x_{1}, y_{1}, z\right)+(1-t) T\left(x_{2}, y_{2}, z\right)+C .
$$

Remark 2.2. Clearly, for each fixed $z \in D$, if $T(\cdot, \cdot, z)$ is $C$-concave on $A \times B$, then $T$ is $C$-concave with respect to its first and second arguments, respectively.

Example 2.1. Let $C \subset Y$ be closed convex pointed cone, $T: A \times B \times D \rightarrow 2^{Y}$ is defined by

$$
T(x, y, z)=f(x)+g(y)+h(z)
$$

where $f$ is $C$-concave on $A$ and $g$ is $C$-concave on $B$. Then, for any $z \in D$, $T(\cdot, \cdot, z)$ is $C$-concave on $A \times B$. Indeed, for any $z \in D,\left(x_{1}, y_{1}\right),\left(x_{2}, y_{2}\right) \in A \times B$ and $t \in[0,1]$,

$$
\begin{aligned}
& T\left(t x_{1}+(1-t) x_{2}, t y_{1}+(1-t) y_{2}, z\right) \\
= & f\left(t x_{1}+(1-t) x_{2}\right)+g\left(t y_{1}+(1-t) y_{2}\right)+h(z) \\
\subset & t f\left(x_{1}\right)+(1-t) f\left(x_{2}\right)+C+t g\left(y_{1}\right)+(1-t) g\left(y_{2}\right)+C+h(z) \\
\subset & t\left(f\left(x_{1}\right)+g\left(y_{1}\right)+h(z)\right)+(1-t)\left(f\left(x_{2}\right)+g\left(y_{2}\right)+h(z)\right)+C \\
= & t T\left(x_{1}, y_{1}, z\right)+(1-t) T\left(x_{2}, y_{2}, z\right)+C .
\end{aligned}
$$

This implies that $T(\cdot, \cdot, z)$ is $C$-concave on $A \times B$. 
Definition 2.4. A topological space $X$ is said to be

(i) connected if, there exist no open subsets $V_{i} \subset X$ with $V_{i} \neq \emptyset$ for $i=1,2$ such that $V_{1} \cup V_{2}=X$ and $V_{1} \cap V_{2}=\emptyset$;

(ii) path-connected if, for each pair of points $x$ and $y$ in $X$, there exists a continuous mapping $\phi:[0,1] \rightarrow X$ such that $\phi(0)=x$ and $\phi(1)=y$.

Lemma 2.1. Suppose that int $C \neq \emptyset$ and int $P \neq \emptyset$. If $F(x, y, A)+C$ and $G(x, y, B)+P$ are convex sets for each $(x, y) \in A \times B$, then

$$
S(F, G)=\bigcup_{(f, g) \in\left(C^{*} \backslash\{0\}\right) \times\left(P^{*} \backslash\{0\}\right)} S(f, g) .
$$

Proof. It is clear that

$$
S(F, G) \supset \bigcup_{(f, g) \in\left(C^{*} \backslash\{0\}\right) \times\left(P^{*} \backslash\{0\}\right)} S(f, g) .
$$

So we only need to show that the converse inclusion of (2.2) holds. For each $(x, y) \in S(F, G)$, we have

$$
\begin{cases}F(x, y, u) \cap(-i n t C)=\emptyset, & \forall u \in A, \\ G(x, y, v) \cap(-i n t P)=\emptyset, & \forall v \in B\end{cases}
$$

and so

$$
\left\{\begin{array}{l}
F(x, y, A) \cap(-i n t C)=\emptyset, \\
G(x, y, B) \cap(-i n t P)=\emptyset .
\end{array}\right.
$$

Now, since $C$ and $P$ are convex pointed cones with

$$
\left\{\begin{array}{l}
(F(x, y, A)+C) \cap(-i n t C)=\emptyset, \\
(G(x, y, B)+P) \cap(-i n t P)=\emptyset .
\end{array}\right.
$$

and $F(x, y, A)+C$ and $G(x, y, B)+P$ are convex sets, by the separation theorem of convex sets, there exists a $(f, g) \in\left(Y^{*} \backslash\{0\}\right) \times\left(Z^{*} \backslash\{0\}\right)$ such that

$$
\inf \{f(F(x, y, u)+c): u \in A, c \in C\} \geq \sup \{f(-c): c \in C\}
$$

and

$$
\inf \{g(G(x, y, v)+p): v \in B, p \in P\} \geq \sup \{f(-p): p \in P\}
$$


From (2.3), we get that $f \in C^{*} \backslash\{0\}$ and

$$
f(F(x, y, u)) \geq 0, \text { for all } u \in A .
$$

Also, by (2.4), we see that $g \in P^{*} \backslash\{0\}$ and

$$
g(G(x, y, v)) \geq 0, \text { for all } v \in B .
$$

It follows that $(x, y) \in S(f, g)$ and so the converse inclusion of (2.2) holds. This completes the proof.

Lemma 2.2. [17]. Let $\left\{X_{i}\right\}_{i \in I}$ be a family of nonempty convex subsets where each $X_{i}$ is contained in a Hausdorff topological vector space $E_{i}$. For each $i \in I$, let $P_{i}: X \rightarrow 2^{X_{i}}$ be a set-valued mapping such that

(i) for each $i \in I, P_{i}(x)$ is convex;

(ii) for each $x \in X, x_{i} \notin P_{i}(x)$;

(iii) for each $y_{i} \in X_{i}, P_{i}^{-1}\left(y_{i}\right)$ is open in $X$;

(iv) for each $i \in I$, there exist a nonempty compact subset $N$ of $X$ and a nonempty compact convex subset $B_{i}$ of $X_{i}$ such that for each $x \in X \backslash N$, there is a $i \in I$ satisfying $P_{i}(x) \cap B_{i} \neq \emptyset$.

Then there exists $x \in X$ such that $P_{i}(x)=\emptyset$ for all $i \in I$.

Lemma 2.3. Let $A \subset X$ and $B \subset E$ be nonempty convex subsets, let $C \subset Y$ and $P \subset Z$ be closed convex pointed cone with int $C \neq \emptyset$ and int $P \neq \emptyset$. Suppose $F: A \times B \times A \rightarrow 2^{Y}$ and $G: A \times B \times B \rightarrow 2^{Z}$ are two set-valued mappings which satisfy conditions:

(i) for each $(x, y) \in A \times B, F(x, y, x) \subset C, F(x, y, y) \subset P$;

(ii) for each $(x, y) \in A \times B, F(x, y, \cdot)$ is $C$-quasiconvex on $A$; for each $(x, y) \in$ $A \times B, G(x, y, \cdot)$ is $P$-quasiconvex on $B$;

(iii) for each $u \in A, F(\cdot, \cdot, u)$ is lower semicontinuous on $A \times B$; for each $v \in B$, $G(\cdot, \cdot, v)$ is lower semicontinuous on $A \times B$;

(iv) there exists nonempty compact convex sets $D_{1} \subset A$ and $D_{2} \subset B$, with each $(x, y) \in A \times B \backslash\left(D_{1} \times D_{2}\right)$, there exist $x^{\prime} \in D_{1}$ such that $F\left(x, y, x^{\prime}\right) \cap-i n t C$ $\neq \emptyset$ or a $y^{\prime} \in D_{2}$ such that $G\left(x, y, y^{\prime}\right) \cap-i n t P \neq \emptyset$.

Then $S(f, g) \neq \emptyset$ for all $(f, g) \in\left(C^{*} \backslash\{0\}\right) \times\left(P^{*} \backslash\{0\}\right)$.

Proof. Define $P_{1}: A \times B \rightarrow 2^{A}$ and $P_{2}: A \times B \rightarrow 2^{B}$, respectively, as follows:

$$
P_{1}(x, y)=\left\{u \in A: f(F(x, y, u)) \not \subset R_{+}^{1}\right\}
$$


and

$$
P_{2}(x, y)=\left\{v \in B: g(G(x, y, v)) \not \subset R_{+}^{1}\right\} .
$$

We prove that $P_{1}$ and $P_{2}$ satisfy all the conditions of Lemma 2.2.

First, we show that $P_{1}(x, y)$ is convex for any $(x, y) \in K$. Suppose that $P_{1}(x, y)$ is not convex. Then there exist $t_{1}, t_{2} \in[0,1]$ with $t_{1}+t_{2}=1, u_{1} \in P_{1}(x, y)$ and $u_{2} \in P_{1}(x, y)$ such that $f\left(F\left(x, y, t_{1} u_{1}+t_{2} u_{2}\right)\right) \subset R_{+}^{1}$. Since $F(x, y, \cdot)$ is $C$ quasiconvex on $A$, by Definition 2.2 (ii), either

$$
F\left(x, y, u_{1}\right) \subset F\left(x, y, t_{1} u_{1}+t_{2} u_{2}\right)+C
$$

or

$$
F\left(x, y, u_{2}\right) \subset F\left(x, y, t_{1} u_{1}+t_{2} u_{2}\right)+C .
$$

It follows that

$$
\text { either } \quad f\left(F\left(x, y, u_{1}\right)\right) \subset R_{+}^{1} \quad \text { or } \quad f\left(F\left(x, y, u_{2}\right)\right) \subset R_{+}^{1},
$$

which is a contradiction with $u_{1} \in P_{1}(x, y)$ and $u_{2} \in P_{1}(x, y)$. Thus, $P_{1}(x, y)$ is convex for any $(x, y) \in A \times B$. Similarly, we can prove that $P_{2}(x, y)$ is convex for any $(x, y) \in A \times B$.

Second, by condition (i), it is easy to see that $x \notin P_{1}(x, y)$ and $y \notin P_{2}(x, y)$ for any $(x, y) \in A \times B$.

Third, we show that $P_{1}^{-1}(u)$ and $P_{2}^{-1}(v)$ are open in $A \times B$ for any $(u, v) \in$ $A \times B$. It is equivalently to show the We only need to prove that the complement sets of $P_{1}^{-1}(u)$ and $P_{1}^{-2}(v)$ are closed. In fact,

$$
\left[P_{1}^{-1}(u)\right]^{c}=\left\{(x, y): f(F(x, y, u)) \subset R_{+}^{1}\right\} .
$$

Let $\left\{\left(x_{n}, y_{n}\right)\right\} \in\left[P_{1}^{-1}(u)\right]^{c}$ with $\left(x_{n}, y_{n}\right) \rightarrow\left(x_{0}, y_{0}\right)$. For each $w_{0} \in F\left(x_{0}, y_{0}, u\right)$, from the lower semicontinuity of $F(\cdot, \cdot, u)$, there exists $w_{n} \in F\left(x_{n}, y_{n}, u\right)$ such that $w_{n} \rightarrow w_{0}$. Since $\left\{\left(x_{n}, y_{n}\right)\right\} \in\left[P_{1}^{-1}(u)\right]^{c}$, we have $f\left(F\left(x_{n}, y_{n}, u\right)\right) \subset R_{+}^{1}$ and so $f\left(w_{n}\right) \geq 0$. Now the continuity of $f$ implies that $f\left(w_{0}\right) \geq 0$. The arbitrariness of $w_{0}$ shows that $f\left(F\left(x_{0}, y_{0}, u\right)\right) \subset R_{+}^{1}$. Thus, $\left(x_{0}, y_{0}\right) \in\left[P_{1}^{-1}(u)\right]^{c}$ and $\left[P_{1}^{-1}(u)\right]^{c}$ is closed. Similarly, we can prove that $\left[P_{2}^{-1}(v)\right]^{c}$ is closed. Therefore, $P_{1}^{-1}(u)$ and $P_{2}^{-1}(v)$ are open in $A \times B$.

Finally, from condition (iv), there exists a nonempty compact set $D_{1} \times D_{2} \subset A \times$ $B$ such that, for any $(x, y) \in K \backslash D$, either $P_{1}(x, y) \cap D_{1} \neq \emptyset$ or $P_{2}(x, y) \cap D_{2} \neq \emptyset$.

Thus, we see that $P_{1}$ and $P_{2}$ satisfy all the conditions of Lemma 2.2 and so there exists $(\bar{x}, \bar{y}) \in A \times B$ such that $P_{i}(\bar{x}, \bar{y})=\emptyset$, i.e.,

$$
\left\{\begin{array}{l}
f(F(\bar{x}, \bar{y}, u)) \geq 0, \quad \forall u \in A, \\
g(G(\bar{x}, \bar{y}, v)) \geq 0, \quad \forall v \in B .
\end{array}\right.
$$

Thus, $S(f, g) \neq \emptyset$. This completes the proof. 
Remark 2.3. Lemma 2.1 is a generalization of Theorem 4.1 in [10] from vector equilibrium problem to symmetric vector equilibrium problem.

Lemma 2.4. Let $A \subset X$ and $B \subset E$ be nonempty closed convex subsets, let $C \subset Y$ and $P \subset Z$ be closed convex pointed cones. Let $F: A \times B \times A \rightarrow 2^{Y}$ and $G: A \times B \times B \rightarrow 2^{Z}$ be set-valued mappings. Suppose that $F(x, y, \cdot)$ is $C$-convex and $G(x, y, \cdot)$ is $P$-convex for any $(x, y) \in A \times B$, then $F(x, y, A)+C$ and $F(x, y, B)+P$ are convex.

Proof. For $t_{1}, t_{2} \in[0,1]$ with $t_{1}+t_{2}=1, y_{1}, y_{2} \in F(x, y, A)$ and $c_{1}, c_{2} \in C$, there exist $u_{1}, u_{2} \in A$ such that $y_{1} \in F\left(x, y, u_{1}\right)$ and $y_{2} \in F\left(x, y, u_{2}\right)$. Since $A$ is convex and $F(x, y, \cdot)$ is $C$-convex, we have

$$
\begin{aligned}
t_{1}\left(y_{1}+c_{1}\right)+t_{2}\left(y_{2}+c_{2}\right) & =\left(t_{1} y_{1}+t_{2} y_{2}\right)+\left(t_{1} c_{1}+t_{2} c_{2}\right) \\
& \subset t_{1} F\left(x, y, u_{1}\right)+t_{2} F\left(x, y, u_{2}\right)+C \\
& \subset F\left(x, y, t_{1} u_{1}+t_{2} u_{2}\right)+C+C \\
& \subset F(x, y, A)+C
\end{aligned}
$$

and so $F(x, y, A)+C$ is convex. Similarly, we can show that $G(x, y, B)+P$ is convex. This completes the proof.

Lemma 2.5. [35]. Suppose that the topological space $X$ is connected and the set-valued mapping $T: X \rightarrow 2^{Y}$ is upper semicontinuous. If for every $x \in X$, the set $T(x)$ is nonempty and connected, then the set $T(X)$ is connected.

We also need the following lemma.

Lemma 2.6. Suppose that $X$ is path-connected and the mapping $T: X \rightarrow Y$ is continuous, then the set $T(X)$ is path-connected.

Proof. For each pair of points $y_{1}$ and $y_{2}$ in $T(X)$, there exist some $x_{1} \in X$ and $x_{2} \in X$ such that $T\left(x_{1}\right)=y_{1}$ and $T\left(x_{2}\right)=y_{2}$. Since $X$ is path-connected, there exists a continuous mapping $\phi:[0,1] \rightarrow X$ such that $\phi(0)=x_{1}$ and $\phi(1)=x_{2}$. Define a mapping $\Psi:[0,1] \rightarrow T(X)$ by $\Psi(t)=T(\Phi(t))$ for all $t \in[0,1]$. By the continuity of $T$ and $\phi$, we know that $\Psi(t)$ is continuous. Moreover, we have $\Psi(0)=T(\Phi(0))=T\left(x_{1}\right)=y_{1}$ and $\Psi(1)=T(\Phi(1))=T\left(x_{2}\right)=y_{1}$. Since $y_{1}$ and $y_{2}$ in $T(X)$ are arbitrary, it follows that the set $T(X)$ is path-connected. This completes the proof.

Lemma 2.7. [6]. Let $X, Y$ be two topological spaces and $T: X \rightarrow 2^{Y}$ be a setvalued mapping. If $Y$ is compact and $T$ is closed, then $T$ is upper semicontinuous. 


\section{Connectedness of the Solution Set}

In this section, we discuss the connectedness of the solution set for the set-valued symmetric vector equilibrium problem. The following Theorem 3.1 establishes the connectedness result of the solution set for the set-valued $S V E P$.

Theorem 3.1. Suppose that the following conditions are satisfied:

(i) for each $(x, y) \in A \times B, F(x, y, x) \subset C, F(x, y, y) \subset P$;

(ii) for each $(x, y, u) \in A \times B \times A, F(\cdot, \cdot, u)$ is $C$-concave on $A \times B, F(x, y, \cdot)$ is $C$-convex and $C$-quasiconvex on $A$; for any $(x, y, v) \in A \times B \times B, G(\cdot, \cdot, v)$ is $P$-concave on $A \times B, F(x, y, \cdot)$ is $P$-convex and $P$-quasiconvex on $B$;

(iii) for each $u \in A, F(\cdot, \cdot, u)$ is lower semicontinuous on $A \times B$; for any $v \in B$, $G(\cdot, \cdot, v)$ is lower semicontinuous on $A \times B$;

(iv) $\{F(x, y, u): x \in A, y \in B, u \in A\}$ is bounded and $\{G(x, y, v): x \in A, y \in$ $B, v \in A\}$ is bounded;

(v) there exist nonempty compact convex sets $D_{1} \subset A$ and $D_{2} \subset B$, for each $(x, y) \in A \times B \backslash\left(D_{1} \times D_{2}\right)$, there exist some $x^{\prime} \in D_{1}$ such that $F\left(x, y, x^{\prime}\right) \cap-i n t C \neq \emptyset$ or some $y^{\prime} \in D_{2}$ such that $G\left(x, y, y^{\prime}\right) \cap-i n t P \neq \emptyset$.

Then $S(F, G)$ is nonempty connected compact.

Proof. From Lemma 2.3, for each $(f, g) \in\left(C^{*} \backslash\{0\}\right) \times\left(P^{*} \backslash\{0\}\right)$, we have $S(f, g) \neq \emptyset$ and so $S(F, G) \neq \emptyset$. Define a set-valued mapping $H:\left(C^{*} \backslash\{0\}\right) \times$ $\left(P^{*} \backslash\{0\}\right) \rightarrow 2^{A \times B}$ by

$$
H(f, g)=S(f, g), \quad \forall(f, g) \in\left(C^{*} \backslash\{0\}\right) \times\left(P^{*} \backslash\{0\}\right) .
$$

We first prove that $H(f, g)$ is convex. In fact, for each $\left(x_{1}, y_{1}\right),\left(x_{2}, y_{2}\right) \in$ $H(f, g)$, the definition of $H$ implies that

$$
\begin{cases}f\left(F\left(x_{1}, y_{1}, u\right)\right) \geq 0, \quad \forall u \in A, \\ g\left(G\left(x_{1}, y_{1}, v\right)\right) \geq 0, \quad \forall v \in B\end{cases}
$$

and

$$
\left\{\begin{array}{l}
f\left(F\left(x_{2}, y_{2}, u\right)\right) \geq 0, \quad \forall u \in A, \\
g\left(G\left(x_{2}, y_{2}, v\right)\right) \geq 0, \quad \forall v \in B .
\end{array}\right.
$$

Since $A$ and $B$ are convex, we known that $A \times B$ is convex. Thus, for any $t_{1}, t_{2} \in[0,1]$ with $t_{1}+t_{2}=1$, we have $t_{1}\left(x_{1}, y_{1}\right)+t_{2}\left(x_{2}, y_{2}\right) \in A \times B$. From 
condition (ii), $F(\cdot, \cdot, u)$ is $C$-concave and $G(\cdot, \cdot, v)$ is $P$-concave on $A \times B$ and so

$$
\left\{\begin{array}{l}
F\left(t_{1} x_{1}+t_{2} x_{2}, t_{1} y_{1}+t_{2} y_{2}, u\right) \subset t_{1} F\left(x_{1}, y_{1}, u\right) \\
\quad+t_{2} F\left(x_{2}, y_{2}, u\right)+C, \quad \forall u \in A \\
G\left(t_{1} x_{1}+t_{2} x_{2}, t_{1} y_{1}+t_{2} y_{2}, v\right) \subset t_{1} G\left(x_{1}, y_{1}, v\right) \\
\quad+t_{2} G\left(x_{2}, y_{2}, v\right)+P, \quad \forall v \in B .
\end{array}\right.
$$

It follows from (3.1), (3.2) and (3.3) that

$$
\begin{cases}f\left(F\left(t_{1} x_{1}+t_{2} x_{2}, t_{1} y_{1}+t_{2} y_{2}, u\right)\right) \geq 0, & \forall u \in A \\ g\left(G\left(t_{1} x_{1}+t_{2} x_{2}, t_{1} y_{1}+t_{2} y_{2}, v\right)\right) \geq 0, & \forall v \in B\end{cases}
$$

This implies that $H(f, g)$ is convex and hence $H(f, g)$ is connected.

Next we prove that $H(\cdot, \cdot)$ is upper semicontinuous on $\left(C^{*} \backslash\{0\}\right) \times\left(P^{*} \backslash\{0\}\right)$. Since $S(f, g) \subset S(F, G) \subset D_{1} \times D_{2}$ and $D_{1} \times D_{2}$ is compact, we only need to show that $H$ is closed by Lemma 2.7. Let $\left\{\left(f_{n}, g_{n}\right),\left(x_{n}, y_{n}\right)\right\} \subset \operatorname{Graph}(H)$ with $\left(f_{n}, g_{n}\right) \rightarrow\left(f_{0}, g_{0}\right)$ and $\left(x_{n}, y_{n}\right) \rightarrow\left(x_{0}, y_{0}\right)$. Then $\left(x_{0}, y_{0}\right) \in D_{1} \times D_{2}$ and

$$
\begin{cases}f_{n}\left(F\left(x_{n}, y_{n}, u\right)\right) \geq 0, & \forall u \in A \\ g_{n}\left(G\left(x_{n}, y_{n}, v\right)\right) \geq 0, & \forall v \in B .\end{cases}
$$

For any $u \in A, v \in B, w_{0} \in F\left(x_{0}, y_{0}, u\right)$ and $\mathrm{z}_{0} \in \mathrm{G}\left(\mathrm{x}_{0}, \mathrm{y}_{0}, \mathrm{v}\right)$, it follows from condition (iii) that there exist $w_{n} \in F\left(x_{n}, y_{n}, u\right)$ and $z_{n} \in G\left(x_{n}, y_{n}, v\right)$ such that $w_{n} \rightarrow w_{0}$ and $z_{n} \rightarrow z_{0}$. From (3.4), we have

$$
f_{n}\left(w_{n}\right) \geq 0, \quad g_{n}\left(z_{n}\right) \geq 0 .
$$

Now the continuity of $\left(f_{0}, g_{0}\right)$ implies that

$$
f_{0}\left(w_{n}\right) \rightarrow f_{0}\left(w_{0}\right), \quad g_{0}\left(z_{n}\right) \rightarrow g_{0}\left(z_{0}\right) .
$$

Moreover, condition (iv) shows that $\left\{w_{n}\right\}$ and $\left\{z_{n}\right\}$ are bounded. It follows from (3.6) that

$$
\lim _{n \rightarrow \infty} f_{n}\left(w_{n}\right)=\lim _{n \rightarrow \infty}\left(f_{n}-f_{0}\right)\left(w_{n}\right)+\lim _{n \rightarrow \infty} f_{0}\left(w_{n}\right)=f_{0}\left(w_{0}\right)
$$

and

$$
\lim _{n \rightarrow \infty} g_{n}\left(z_{n}\right)=\lim _{n \rightarrow \infty}\left(g_{n}-g_{0}\right)\left(z_{n}\right)+\lim _{n \rightarrow \infty} g_{0}\left(z_{n}\right)=g_{0}\left(z_{0}\right) .
$$

By (3.5), we know that $f_{0}\left(w_{0}\right) \geq 0$ and $g_{0}\left(z_{0}\right) \geq 0$. Since $w_{0}$ and $z_{0}$ are arbitrary,

$$
\begin{cases}f_{0}\left(F\left(x_{0}, y_{0}, u\right)\right) \geq 0, & \forall u \in A, \\ g_{0}\left(G\left(x_{0}, y_{0}, v\right)\right) \geq 0, & \forall v \in B .\end{cases}
$$


This implies that $\left(x_{0}, y_{0}\right) \in H\left(f_{0}, g_{0}\right)$ and so $H$ is closed. Therefore, $H$ is upper semicontinuous on $\left(C^{*} \backslash\{0\}\right) \times\left(P^{*} \backslash\{0\}\right)$. Moreover, from condition (ii) and Lemma 2.1 and 2.4 , we have

$$
S(F, G)=\bigcup_{(f, g) \in\left\{C^{*} \backslash\{0\}\right\} \times\left\{P^{*} \backslash\{0\}\right\}} S(f, g),
$$

and so Lemma 2.5 implies that $S(F, G)$ is connected.

Finally, we show that $S(F, G)$ is compact. Let $\left(x_{n}, y_{n}\right) \in S(F, G)$ with $\left(x_{n}, y_{n}\right) \rightarrow\left(x_{0}, y_{0}\right)$. We claim that $\left(x_{0}, y_{0}\right) \in S(F, G)$. If $\left(x_{0}, y_{0}\right) \notin S(F, G)$, then there exist some $u_{0} \in A$ such that

$$
F\left(x_{0}, y_{0}, u_{0}\right) \cap-i n t C \neq \emptyset
$$

or some $v_{0} \in B$ such that

$$
G\left(x_{0}, y_{0}, v_{0}\right) \cap-i n t P \neq \emptyset .
$$

If (3.7) holds, then there exists some $d_{0} \in F\left(x_{0}, y_{0}, u_{0}\right)$ such that

$$
d_{0} \in-i n t C \text {. }
$$

By the lower semicontinuity of $F\left(\cdot, \cdot, u_{0}\right)$, there exists a sequence $\left\{d_{n}\right\} \in F\left(x_{n}, y_{n}, u_{0}\right)$ such that $d_{n} \rightarrow d_{0}$. Since $\left(x_{n}, y_{n}\right) \in S(F, G)$, we know that $d_{n} \notin-i n t C$ and so $d_{n} \in Y \backslash\{-i n t C\}$. Clearly, $Y \backslash\{-i n t C\}$ is closed. Since $d_{n} \rightarrow d_{0}$, we have $d_{0} \in Y \backslash\{-i n t C\}$ and so $d_{0} \notin-i n t C$, which is a contradiction with (3.9). Thus, $\left(x_{0}, y_{0}\right) \in S(F, G)$. If (3.8) holds, by using the similar way, we can show that $\left(x_{0}, y_{0}\right) \in S(F, G)$. Therefore, $S(F, G)$ is closed. Now the compactness of $D_{1} \times D_{2}$ and $S(F, G) \subset D_{1} \times D_{2}$ imply that $S(F, G)$ is compact. This completes the proof.

Remark 3.1. (i) If $A \times B$ is compact in $X \times E$, then condition (v) in Theorem 3.1 can be removed; (ii) Theorem 3.1 generalizes Theorem 4.5 of [24] from the singlevalued vector equilibrium problem to the set-valued symmetric vector equilibrium problem; (iii) Theorem 3.1 generalizes Theorem 5.1 of [10] from the set-valued vector equilibrium problem to the set-valued symmetric vector equilibrium problem; (iv) Theorem 3.1 also generalizes the corresponding connectedness results in [14] and [33].

We give the following example to illustrate Theorem 3.1.

Example 3.1. Let $A=B=[0,2]$ and $C=P=R_{+}^{1}$. Let $F(x, y, u)=$ $y+u^{2}-x^{2}$ for all $(x, y, u) \in A \times B \times A$ and $G \equiv 0$. Then $F$ and $G$ satisfy all the conditions of Theorem 3.1. Further, it is easy to see that

$$
S(F, G)=\left\{(x, y):(x, y) \in[0,2] \times[0,2], y \geq x^{2}\right\} .
$$

Clearly, $S(F, G)$ is nonempty connected compact. 


\section{Path-connectedness of the Solution Set}

In this section, we establish a path-connectedness result of the solution set for the symmetric vector equilibrium problem under some suitable assumptions.

Theorem 4.1. Suppose that all the conditions of Theorem 3.1 are satisfied. Moreover, suppose that $F$ and $G$ satisfy the following monotonicity assumption $(\Sigma)$ : for any $\left(x_{1}, y_{1}\right),\left(x_{2}, y_{2}\right) \in A \times B$ with $\left(x_{1}, y_{1}\right) \neq\left(x_{2}, y_{2}\right)$,

$$
\left(F\left(x_{1}, y_{1}, x_{2}\right) \cap(-i n t C)\right) \bigcup\left(G\left(x_{1}, y_{1}, y_{2}\right) \cap(-i n t P)\right)=\emptyset
$$

implies that

$$
\Rightarrow\left(F\left(x_{2}, y_{2}, x_{1}\right) \cap(-i n t C)\right) \bigcup\left(G\left(x_{2}, y_{2}, y_{1}\right) \cap(-i n t P)\right) \neq \emptyset .
$$

Then $S(F, G)$ is nonempty path-connected compact.

Proof. We first proof that for each $(f, g) \in\left(C^{*} \backslash\{0\}\right) \times\left(P^{*} \backslash\{0\}\right), S(f, g)$ has exactly one solution in $A \times B$. Let $\left(x_{1}, y_{1}\right) \in S(f, g)$ and $\left(x_{2}, y_{2}\right) \in S(f, g)$ with $\left(x_{1}, y_{1}\right) \neq\left(x_{2}, y_{2}\right)$. Then

$$
\left\{\begin{array}{l}
f\left(F\left(x_{1}, y_{1}, u\right)\right) \geq 0, \quad \forall u \in A, \\
g\left(G\left(x_{1}, y_{1}, v\right)\right) \geq 0, \quad \forall v \in B
\end{array}\right.
$$

and

$$
\left\{\begin{array}{l}
f\left(F\left(x_{2}, y_{2}, u\right)\right) \geq 0, \quad \forall u \in A, \\
g\left(G\left(x_{2}, y_{2}, v\right)\right) \geq 0, \quad \forall v \in B .
\end{array}\right.
$$

Taking $u=x_{2}, v=y_{2}$ in (4.1) and $u=x_{1}, v=y_{1}$ in (4.2), respectively, we have

$$
\left\{\begin{array}{l}
f\left(F\left(x_{1}, y_{1}, x_{2}\right)\right) \geq 0 \\
g\left(G\left(x_{1}, y_{1}, y_{2}\right)\right) \geq 0
\end{array}\right.
$$

and

$$
\left\{\begin{array}{l}
f\left(F\left(x_{2}, y_{2}, x_{1}\right)\right) \geq 0 \\
g\left(G\left(x_{2}, y_{2}, y_{1}\right)\right) \geq 0
\end{array}\right.
$$

It follows from (4.3) that

$$
\left\{\begin{array}{l}
F\left(x_{1}, y_{1}, x_{2}\right) \cap(-i n t C)=\emptyset \\
G\left(x_{1}, y_{1}, y_{2}\right) \cap(-i n t P)=\emptyset
\end{array}\right.
$$


By (4.5) and the monotonicity assumption $(\Sigma)$, either $F\left(x_{2}, y_{2}, x_{1}\right) \cap(-i n t C) \neq \emptyset$ or $G\left(x_{2}, y_{2}, y_{1}\right) \cap(-i n t P) \neq \emptyset$. This is a contradiction with (4.4) and hence $S(f, g)$ is single-valued.

Moreover, from the proof of Theorem 3.1, we see that $H(\cdot, \cdot)=S(\cdot, \cdot)$ is a single-valued upper semicontinuous mapping on $\left(C^{*} \backslash\{0\}\right) \times\left(P^{*} \backslash\{0\}\right)$ and hence continuous. Also, the convexity of $\left(C^{*} \backslash\{0\}\right) \times\left(P^{*} \backslash\{0\}\right)$ implies that it is a pathconnected set. It follows from Lemma 2.6 that $S(F, G)=H\left(\left(C^{*} \backslash\{0\}\right) \times\left(P^{*} \backslash\{0\}\right)\right)$ is also path-connected. This completes the proof.

Remark 4.1. Theorem 4.1 generalizes Theorem 4.2 (i) of [32] from the singlevalued vector variational inequality to the set-valued symmetric vector equilibrium problem.

The following Example 4.1 shows that assumption $(\Sigma)$ can be satisfied.

Example 4.1. Let $A=B=R, C=P=R_{+}^{1}, F(x, y, u)=\left(y^{2}+1\right)(u-x)$ for all $(x, y, u) \in A \times B \times A$ and $G(x, y, v)=\left(x^{2}+1\right)(v-y)$ for all $(x, y, v) \in$ $A \times B \times B$. Then $F$ and $G$ satisfy the monotonicity assumption $(\Sigma)$. Indeed, for any $\left(x_{1}, y_{1}\right),\left(x_{2}, y_{2}\right) \in A \times B,\left(x_{1}, y_{1}\right) \neq\left(x_{2}, y_{2}\right)$, if

$$
\left\{\begin{array}{l}
F\left(x_{1}, y_{1}, x_{2}\right)=\left(y_{1}^{2}+1\right)\left(x_{2}-x_{1}\right) \geq 0 \\
G\left(x_{1}, y_{1}, y_{2}\right)=\left(x_{1}^{2}+1\right)\left(y_{2}-y_{1}\right) \geq 0
\end{array}\right.
$$

then we have $x_{2}>x_{1}$ and $y_{2}>y_{1}$. It follows that $F\left(x_{2}, y_{2}, x_{1}\right)=\left(y_{2}^{2}+1\right)\left(x_{1}-\right.$ $\left.x_{2}\right)<0$ and $G\left(x_{2}, y_{2}, y_{1}\right)=\left(x_{2}^{2}+1\right)\left(y_{1}-y_{2}\right)<0$. Therefore, $F$ and $G$ satisfy the monotonicity assumption $(\Sigma)$.

\section{REFERENCES}

1. Q. H. Ansari, W. Oettli and D. Schiager, A generalization of vector equilibria, Math. Meth. Oper. Res., 46 (1997), 147-527.

2. Q. H. Ansari and J. C. Yao, An existence result for the generalized vector equilibrium, Appl. Math. Lett., 12 (1999), 53-56.

3. Q. H. Ansari, S. Schaible and J. C. Yao, The system of vector equilibrium problems andits applications, Optim. Theory Appl., 107 (2000), 547-557.

4. Q. H. Ansari, W. K. Chan and X. Q. Yang, The system of vector quasi-equilibrium problems with applications, J. Global Optim., 29 (2004), 45-57.

5. Q. H. Ansari, W. K. Chan and X. Q. Yang, Weighted quasi-variational inequalities and constrained Nash equilibrium problems, Taiwanese J. Math., 10 (2006), 361-380.

6. J. P. Aubin and I. Ekeland, Applied Nonlinear Analysis, Wiley, New York, 1984. 
7. E. Blum and W. Oettli, From optimization and variational inequalities to equilibrium problems, Math. Stud., 63 (1994), 123-145.

8. M. Bianchi and S. Schaible, Generalized monotone bifunctions and equilibrium problems, J. Optim. Theory Appl., 90 (1996), 31-43.

9. M. Bianchi, N. Hadjisavvas and S. Schaible, Vector equilibrium problems with generalized monotone bifunctions, J. Optim. Theory Appl., 92 (1997), 527-542.

10. B. Chen, X. H. Gong and S. M. Yuan, Connectedness and compactness of weak efficient solutions for set-valued vector equilibrium problems, J. Inequal. Appl., 2008 (2008), Article ID 581849, 15 pages.

11. G. Y. Chen and G. M. Cheng, Vector variational inequalities and vector optimization, in: Lecture Notes in Economics and Mathematical Systems, Vol. 258, pp. 408-416, Springer, Heidelberg, 1987.

12. G. Y. Chen, X. X. Huang and X. Q. Yang, Vector Optimization: Set-Valued and Variational Analysis, Springer, Berlin, 2005.

13. G. Y. Chen, X. Q. Yang and H. Yu, A nonlinear scalarization function and generalized quasi-vector equilibrium problem, J. Global Optim., 32 (2005), 451-466.

14. Y. H. Cheng, On the connectedness of the solution set for the weak vector variational inequality, J. Math. Anal. Appl., 260 (2001), 1-5.

15. X. P. Ding, J. C. Yao and L. J. Lin, Solutions of system of generalized vector quasiequilibrium problems in locally $G$-convex uniform spaces, J. Math. Anal. Appl., 298 (2004), 389-410.

16. G. Debreu, A social equilibrium existence theorem,Proc. Nat. Acad. Sci. USA, 38 (1952), 886-893.

17. P. Deguire, K. K. Tan and G. X. Z. Yuan, The study of maximal elements, fixed point for Ls-majorized mappings and the quasi-variational inequalities in product spaces, Nonlinear Anal., 37 (1999), 933-951.

18. J. Y. Fu, Symmetric vector quasi-equilibrium problems, J. Math. Anal. Appl., 285 (2003), 708-713.

19. M. Fakhar and J. Zafarani, Generalized symmetric vector quasiequilibrium problems, J. Optim. Theory Appl., 136 (2008), 397-409.

20. Ali P. Farajzadeh, On the symmetric vector quasi-equilibrium problems, J. Math. Anal. Appl., 322 (2006), 1099-1110.

21. F. Giannessi, Theorem of the alternative, quadratic programs, and complementarity problems. In: R.W. Cottle, F. Giannessi, J.L. Lions, (Eds.) Variational Inequalities and Complementarity Problems, pp. 151-186. Wiley, New York, 1980.

22. F. Giannessi (Ed.), Vector Variational Inequalities and Vector Equilibrium, Kluwer Academic Publishers, Dordrecht, Boston, London, 2000.

23. X. H. Gong, Symmetric strong vector quasi-equilibrium problems, Math. Meth. Oper. Res., 65 (2007), 305-314. 
24. X. H. Gong, Connectedness of the solution sets and scalarization for vector equilibrium problems, J. Optim. Theory Appl., 133 (2007), 151-161.

25. X. H. Gong and J. C. Yao, Connectedness of the Set of Efficient Solutions for Generalized Systems, J. Optim. Theory Appl., 138 (2008), 189-196.

26. N. J. Huang, J. Li and H. B. Thompson, Implicit vector equilibrium problems with applications, Math. Comput. Modelling., 37 (2003), 1343-1356.

27. N. J. Huang and C. J. Gao, Some generalized vector variational inequalities and complementarity problems for multivalued mappings, Appl. Math. Lett., 16 (2003), 1003-1010.

28. N. J. Huang, J. Li and J. C. Yao, Gap Functions and existence of solutions for a system of vector equilibrium problems, J. Optim. Theory Appl., 133 (2007), 201-212.

29. J. Li, N. J. Huang and J. K. Kim, On implicit vector equilibrium problems, J. Math. Anal. Appl., 283 (2003), 501-512.

30. L. J. Lin, System of generalized vector quasi-equilibrium problems with applications to fixed point theorems for a family of nonexpansive multivalued maps, J. Global Optim., 34 (2006), 15-32.

31. L. J. Lin and H. W. Hsu, Existence theorems for systems of generalized vector quasiequilibrium problems and optimization problems, J. Global Optim., 37 (2007), 195-213.

32. G. M. Lee, D. S. Kim, B. S. Lee and N. D. Yen, Vector variational inequality as a tool for studying vector optimization problems, Nonlinear Anal., 34 (1998), 745-765.

33. G. M. Lee and I. J. Bu, On solution sets for affine vector variational inequality, Nonlinear Anal., 63 (2005), 1847-1855.

34. X. J. Long, N. J. Huang and K. L. Teo, Existence and stability of solutions for generalized strong vector quasi-equilibrium problem, Math. Comput. Modelling, 47 (2008), 445-451.

35. A. R. Warburton, Quasiconcave vector maximization: connectedness of the sets of Pareto-optimal and weak Pareto-optimal alternatives, J. Optim. Theory Appl., 40 (1983), 537-557.

36. X. Q. Yang, Vector variational inequality and its duality, Nonlinear Anal. Theory Methods Appl., 21 (1993), 869-877.

Ren-you Zhong and Nan-jing Huang

Department of Mathematics,

Sichuan University,

Chengdu, Sichuan 610064,

P. R. China 
Mu-Ming Wong

Department of Applied Mathematics,

Chung Yuan Christian University,

Chung-Li 32023, Taiwan

E-mail: mmwong@cycu.edu.tw 